\title{
Delayed Bit Interleaved Coded Sparse Code Multiple Access
}

\author{
Zeina Mheich, Zilong Liu, Pei Xiao and Amine Maaref
}

\begin{abstract}
Targeting to provide reliable short-packet communications with tens of bits in machine-type networks, we investigate a novel sparse code multiple access (SCMA) scheme called delayed bit-interleaved coded SCMA (DBIC-SCMA). At the transmitter side, a delay module is introduced between each channel encoder and SCMA mapper such that data bits from different channel codewords can be mapped to an identical SCMA codeword. We present the main components and principles for the transmitter of DBIC-SCMA, followed by a predecoding assisted receiver design which exploits systematic and rate-adaptive properties of certain channel codes. Our simulation results show that the proposed DBIC-SCMA leads to significant improvements in error rate performance over the classical BICSCMA scheme.
\end{abstract}

Index Terms-NOMA, SCMA, BICM, DBICM, LDPC, Turbo.

\section{INTRODUCTION}

Bit-interleaved coded modulation (BICM) with Gray labeling can potentially approach the capacity of coded modulation (CM) systems with lower implementation complexity compared to its counterparts such as trellis-coded modulation or multi-level coded modulation. In BICM, a bit interleaver is inserted between the channel encoder and the modulation mapper to eliminate the correlation of coded bits mapped to the same modulation symbols. Recently, a variant of BICM called delayed BICM (DBICM) has been proposed [1]-[3]. DBICM outperforms the traditional BICM for certain constellations by taking advantage of the interblock memory [4] among multiple codewords [5]. This is achieved by introducing a delay module between the channel encoder and the modulation mapper such that data bits from different codewords can be mapped to an identical symbol. It is shown in [5] that DBICM can achieve higher capacity than BICM at the expense of a slight increase in latency. In particular, DBICM can achieve significant gains over BICM for non-standard constellations where Gray labeling may not be applicable ${ }^{1}$. Simulation results in [5] show

Copyright (c) 2015 IEEE. Personal use of this material is permitted. However, permission to use this material for any other purposes must be obtained from the IEEE by sending a request to pubs-permissions@ieee.org.

This work was supported in part by a research grant from Huawei Technologies Canada Co. Ltd. It was also supported in part by the UK Engineering and Physical Sciences Research Council (EPSRC) under grant number EP/P03456X/1.

Zeina Mheich and Pei Xiao are with the 5G Innovation Centre, Institute for Communication Systems, University of Surrey, U.K. (e-mail: \{z.mheich,p.xiao\}@surrey.ac.uk). Zilong Liu is with the School of Computer Science and Electrical Engineering, University of Essex, U.K. (e-mail: zilong.liu@essex.ac.uk).

Amine Maaref is with Huawei Technologies Canada Co. Ltd., Ottawa, ON, Canada. (e-mail: Amine.Maaref@huawei.com).

${ }^{1}$ These constellations are widely used in optical communications as stated in [5]. that there is significant gain for half-16-QAM compared to marginal gain for standard 16-QAM.

Inspired by the above observation, we apply DBICM to SCMA whose constellations are mostly non-standard [6], [7]. SCMA is an emerging code-domain non-orthogonal multiple access (NOMA) paradigm which enables overloaded multiuser communications [8]. In NOMA schemes, the same resource, e.g. time/frequency/code, is used by multiple users, whereas conventional OMA schemes, e.g. time/frequency/code-division multiple access, rely on orthogonality to avoid interference between users [9]. As a result, NOMA has emerged as a promising technique to support efficient information exchange of massive communication devices in machine-type networks. Although the design of coded NOMA systems has been investigated in several works (e.g. [10], [11], [12]), DBICSCMA has not been reported in the literature, to the best of our knowledge. We consider short channel codes which are of particular interest to machine-type communications and ultra-reliable and low-latency communications (URLLC). A plus point of DBIC-SCMA is that it does not prevent other users from using BIC-SCMA in the same transmission. The main contributions and findings of this work are summarized as follows:

1) We show that when an SCMA user employs DBICSCMA scheme, the gain is not limited to this specific user but also beneficial to the remaining ones which share the same resources. It is found that largest gain is attained when all the SCMA users employ DBIC-SCMA scheme.

2) We propose a novel receiver structure for DBIC-SCMA by exploiting systematic and rate-adaptive properties of certain low-density parity check (LDPC) codes in 5G New Radio (NR). The main idea is to perform LDPC pre-decoding when some partially received signals are received. Significant error rate performance gains are observed for the proposed receiver over the ones for DBIC-SCMA without pre-decoding and BIC-SCMA.

The rest of the paper is organised as follows. Section II-A introduces the transmitter structure and the proposed predecoding based receiver for DBIC-SCMA. Section III presents error rate performance using 5G compliant LDPC codes. Finally, Section IV concludes the paper. The key notations used in the paper are summarized in Table I. 
Table I

SUMMARY OF KEY NOTATIONS.

\begin{tabular}{ll}
\hline \hline Notation & Meaning \\
\hline$J$ & Number of users \\
$M$ & Number of resources \\
$u_{j}$ & SCMA codebook size for each user \\
$\mathbf{C}_{j}$ & The $j$ th SCMA user \\
$R$ & SCMA codebook of user $u_{j}$ \\
$\mathbf{x}_{j}$ & Channel code rate \\
$\mathbf{h}_{j}$ & Transmitted SCMA codeword for user $u_{j}$ \\
& The fading channel vector between user $u_{j}$ \\
$t$ & and the receiver \\
$\mathbf{c}_{t}^{j}$ & Time index \\
$n$ & The channel codeword of user $u_{j}$ \\
$\pi_{j}$ & The length of $\mathbf{c}_{t}^{j}$ \\
$\mathbf{c}_{t}^{j}(i)$ & Interleaver used by $u_{j}$ \\
$T_{i}$ & The $i$ th sub-block of codeword $\mathbf{c}_{t}^{j}$ \\
$m_{r_{k} \rightarrow u_{j}}^{(\tau)}(\mathbf{x})$ & Delay attributed to the sub-block $\mathbf{c}_{t}^{j}(i)$ \\
$m_{u_{j} \rightarrow r_{k}}^{(\tau)}(\mathbf{x})$ & The belief message from resource $r_{k}$ to user $u_{j}$ at the \\
& $\tau$ th MPA iteration about the codeword $\mathbf{x}$. \\
$\mathcal{I}_{r}(k)$ & The belief message from user $u_{j}$ to resource $r_{k}$ at the \\
$\mathcal{I}_{u}(j)$ & $\tau$ th MPA iteration about the codeword $\mathbf{x}$. \\
\hline \hline
\end{tabular}

\section{Delayed Bit-interleaved Coded SCMA}

\section{A. SCMA system model}

We consider an uplink SCMA system where $J$ users share $K$ resource nodes with $K<J$. In practice, SCMA is implemented using orthogonal frequency-division multiplexing (OFDM) where every resource node refers to a subcarrier channel. Each user is assigned with a multi-dimensional SCMA codebook consisting of $M$ codewords. Every codeword is sparse and will be sent over $K$ orthogonal resources. At the transmitter of user $u_{j}$, the SCMA mapper maps each vector of $\log _{2}(M)$ coded bits to a multi-dimensional codeword $\mathbf{x}_{j}$ selected from the corresponding SCMA codebook. The sparsity of the SCMA codewords implies a few number of users (less than $J$ ) colliding over the same resource. This enables the use of message passing algorithm (MPA) for multiuser detection performance close to maximum likelihood receiver. It is noted that every SCMA system can be represented by a factor graph with two sets of nodes: user nodes and resource nodes. A user node is connected to a resource node by an edge in the factor graph if and only if such a resource node is used by this specific SCMA user with active transmission.

\section{B. DBIC-SCMA transmitter}

A DBIC-SCMA transmitter structure is shown in the upper part of Fig. 1. For each user $u_{j}$, the channel coding module encodes a packet $\mathbf{b}_{t}^{j}$ of $k$ bits arriving at time slot $t$ into a codeword of length $n$, where $j \in \mathcal{J}=\{1,2, \cdots, J\}^{2}$. The code rate is defined as $R=k / n$. After passing through the interleaver $\pi_{j}$, the interleaved codeword $\mathbf{c}_{t}^{j}$ is split by the "serial to parallel" module into $m$ sub-blocks

$$
\mathbf{c}_{t}^{j}(i)=\left[c_{t, i \cdot n / m+0}^{j}, c_{t, i \cdot n / m+1}^{j}, \cdots, c_{t, i \cdot n / m+n / m-1}^{j}\right]^{T},
$$

\footnotetext{
${ }^{2}$ For simplicity of notation, we assume that the same channel code is applied to all SCMA users.
}

where $m=\log _{2}(M), i \in\{0,1, \cdots, m-1\}$ and $M$ denotes the size of the SCMA codebook for each user. A delay module $Z^{-T_{i}}$ is then applied to each sub-block $\mathbf{c}_{t}^{j}(i)$ which introduces a delay of $T_{i}$. Each delay unit is equal to a duration of $n / m$ bits. The SCMA mapper ${ }^{3}$ takes one input bit from each delayed sub-block and then maps the resulting $m$ bits to an SCMA codeword $\mathbf{x}_{j}$ occupying $K$ resource nodes. The lower part of Fig. 1 presents an example of two delayed bit streams involving four codewords, where $T_{0}=0$ and $T_{1}=1$. For ease of presentation, the following notations are used in the lower part of Fig. 1:

$$
\begin{array}{ll}
A_{0} \triangleq \mathbf{c}_{t}^{j}(0), & A_{1} \triangleq \mathbf{c}_{t}^{j}(1), \\
B_{0} \triangleq \mathbf{c}_{t+1}^{j}(0), & B_{1} \triangleq \mathbf{c}_{t+1}^{j}(1), \\
C_{0} \triangleq \mathbf{c}_{t+2}^{j}(0), & C_{1} \triangleq \mathbf{c}_{t+2}^{j}(1), \\
D_{0} \triangleq \mathbf{c}_{t+3}^{j}(0) . &
\end{array}
$$

Moreover, in the sequel, the concatenation of $A_{0}$ and $A_{1}$ will be called codeword $A$. Similarly, we have codeword $B$. The lower part of Fig. 1 shows that each SCMA codeword is associated with a vector of two bits $\left(d_{0}, d_{1}\right)$ which are from two different codewords, i.e., the bit $d_{0}$ carries a coded bit from the current codeword while $d_{1}$ carries a coded bit from the previous codeword. In this work, we use LDPC codes in $5 \mathrm{G}$ NR as the channel code.

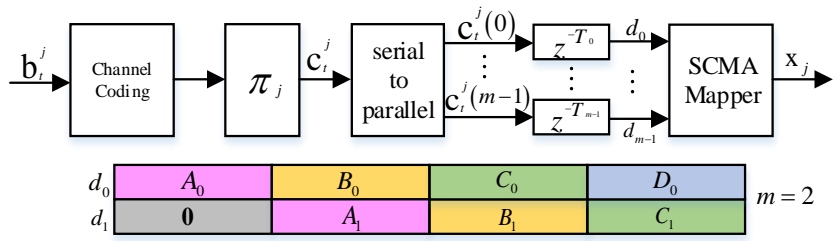

Figure 1. A DBIC-SCMA transmitter with an illustration of $m=2$ delayed bit streams for SCMA encoding $(M=4, t=0)$. Natural block interleaving is introduced as the SCMA mapper takes $m$ delayed bit streams slice by slice.

\section{DBIC-SCMA receiver design}

The received signal $\mathbf{y}$ can be written as

$$
\mathbf{y}=\sum_{j=1}^{J} \operatorname{diag}\left(\mathbf{h}_{j}\right) \mathbf{x}_{j}+\mathbf{n},
$$

where $\mathbf{h}_{j}=\left(h_{1}^{j}, \cdots, h_{K}^{j}\right)^{T}$, where $h_{k}^{j} \sim \mathcal{C N}(0,1)$, denotes the channel vector between user $u_{j}$ and the receiver, and $\mathbf{n}=\left(n_{1}, \cdots, n_{K}\right)^{T}$ with $n_{k} \sim \mathcal{C N}\left(0, N_{0}\right)$ denotes the complex additive white Gaussian noise vector. By exploiting the sparsity of SCMA codebooks, efficient MPA can be applied which leads to error rate performance approaching to that of the maximum likelihood detector. The complexity of MPA is $\mathcal{O}\left(M^{d_{f}}\right)$ where $d_{f}$ is the number of users sharing an identical resource node. MPA iteratively computes an approximation of the maximum a posteriori value by exchanging belief messages between users and resource nodes in the Tanner graph associated to the SCMA system [8]. Let $m_{u_{j} \rightarrow r_{k}}^{(\tau)}(\mathbf{x})$ denote the belief message associated with the SCMA codeword $\mathrm{x}$ which is transmitted from user node $u_{j}$, to resource node $r_{k}$ at the

${ }^{3}$ i.e., SCMA encoder which is equivalent to the modulation mapper in DBICM. 
$\tau$-th iteration of the MPA. Similarly, denote by $m_{r_{k} \rightarrow u_{j}}^{(\tau)}(\mathbf{x})$ the belief message from resource node $r_{k}$ to user node $u_{j}$. $m_{r_{k} \rightarrow u_{j}}^{(\tau)}(\mathbf{x})$ and $m_{u_{j} \rightarrow r_{k}}^{(\tau)}(\mathbf{x})$ are given by [13]:

$$
\begin{aligned}
m_{r_{k} \rightarrow u_{j}}^{(\tau)}(\mathbf{x})= & \sum_{\substack{\mathbf{x}_{j}=\mathbf{x} \\
i \in \mathcal{I}^{\prime}(k) \backslash\{j\} \\
\mathbf{x}_{i} \in \mathbf{C}_{i}}} \frac{1}{\pi N_{0}} e^{-\frac{\left|y_{k}-\sum_{i \in \mathcal{I}_{r}(k)} h_{k}^{i} x_{k i}\right|^{2}}{N_{0}}} \\
& \cdot \prod_{i \in \mathcal{I}_{r}(k) \backslash\{j\}} m_{u_{i} \rightarrow r_{k}}^{(\tau-1)}\left(\mathbf{x}_{i}\right),
\end{aligned}
$$

and

$$
m_{u_{j} \rightarrow r_{k}}^{(\tau)}(\mathbf{x})=\alpha_{j} \cdot \prod_{\ell \in \mathcal{I}_{u}(j) \backslash\{k\}} m_{r_{\ell} \rightarrow u_{j}}^{(\tau)}(\mathbf{x}),
$$

respectively, where $\mathbf{C}_{i}$ denotes the SCMA codebook of user $u_{i}, \alpha_{j}$ is a normalization factor, $\mathcal{I}_{r}(k)$ and $\mathcal{I}_{u}(j)$ are the set of user indices sharing resource node $r_{k}$ and the set of resource indices used by user $u_{j}$, respectively. At the initial iteration $\tau=0$, we assume that

$$
m_{u_{j} \rightarrow r_{k}}^{(0)}(\mathbf{x})=1 / M, \quad \forall j \in \mathcal{J}, k \in \mathcal{I}_{u}(j) .
$$

MPA terminates, when a predefined maximum number of iterations is reached or when the convergence of the messages is achieved. The receiver of BIC-SCMA is composed of the SCMA detector (MPA) followed by the de-interleavers and the channel decoders. A turbo-like receiver may be implemented to enhance the error rate performance of BIC-SCMA by passing a priori messages from channel decoders to the SCMA detector [12]. In the following sub-section, we propose a novel DBIC-SCMA receiver which explores some excellent structures of certain channel codes.

1) Proposed Pre-decoding Assisted Receiver: The proposed receiver for DBIC-SCMA carries out pre-decoding based on partially received signals. Hence, it requires systematic and rate-adaptive channel code with $R \leq 1 / 2$, so that a codeword $\mathbf{c}$ can be split into two parts: $\mathbf{c}=\left[\mathbf{c}_{s}, \mathbf{c}_{p}\right]$, where $\mathbf{c}_{s}$ and $\mathbf{c}_{p}$ are the parts carrying the information bits and parity bits, respectively. By noting the fact that natural block interleaving is introduced as the SCMA mapper takes $m$ delayed bit streams slice by slice, the interleaver $\pi_{j}$ in Fig. 1 may be omitted. Over the time window of each sub-block group ${ }^{4}$, the received signals carry information related to delayed and non-delayed messages from multiple codewords. After multiuser detection using MPA, the receiver can decode both non-delayed and delayed messages utilizing the fact that the channel code is systematic and rate-adaptive. Assuming that all sub-block groups have the same length, $n_{g}=n / m$ c.u., the receiver can decode the non-delayed message with a rate equal to $k / n_{g}$. For the delayed messages, the receiver can decode them using current and past observations. When the decoding of one message (or more) is successful, MPA can be re-run to take advantage of the successfully decoded bit to improve the logarithm likelihood ratios (LLR) of the remaining bits.

Let us revisit the example shown in the lower part of Fig. 1. If the codeword $A$ is decoded correctly in the first sub-block group (i.e., $A_{0}$ occupying channel uses from 1 to

\footnotetext{
${ }^{4}$ In Figure 1, a sub-block group, e.g., $A_{0}$ or $A_{1}$, occupies $n / 2$ channel uses (c.u.)
}

$n / 2$ ), then the receiver can obtain the remaining bits of the codeword transmitted in the second sub-block group (i.e., $A_{1}$ occupying channel uses from $n / 2+1$ to $n$ ) by re-encoding the estimated packet $\hat{\mathbf{b}}_{t}$. Upon obtaining the whole codeword $A$, the receiver may remove the interference to $B_{0}$ caused by $A_{1}$. More specifically, when the first bit $d_{0}$ of vector $\left(d_{0}, d_{1}\right)$ is known, one may use "MPA with partially known messages (MPA-PNM)", i.e., the effective codebook size can be reduced to 2 from 4 . On the other hand, if $A_{0}$ is not decoded correctly, the channel decoder can feedback LLRs of the bits associated with $A_{1}$ as extrinsic information to be used by MPA in the next decoding iteration [1].

In order to implement MPA-PNM, the DBIC-SCMA receiver requires a feedback from the channel decoder to the MPA. The feedback carries a priori LLRs of the bits $\mathbf{d}_{j}=$ $\left(d_{0}^{j}, d_{1}^{j}, \cdots, d_{m-1}^{j}\right)$ to decode the SCMA codeword of user $u_{j}$. Assume that $\tilde{m}_{j}$ bits of $\mathbf{d}_{j}$ are known because they belong to codewords which are decoded correctly by the receiver ${ }^{5}$. Let $\mathcal{D}_{j}$ denotes the set of these $\tilde{m}_{j}$ bits. As a result, the receiver can determine the effective codebook $\tilde{\mathbf{C}}_{i}$ for user $u_{i}$ which consists of all codewords in $\mathbf{C}_{i}$ with bit vectors satisfying $\mathcal{D}_{j}$. Thus, the effective codebook size reduces to $\tilde{M}_{j}=2^{m-\tilde{m}_{j}}$. Then, the message update in (3) will use $\tilde{\mathbf{C}}_{i}$ instead of $\mathbf{C}_{i}$ which reduces the complexity of MPA:

$$
\begin{aligned}
m_{r_{k} \rightarrow u_{j}}^{(\tau)}(\mathbf{x})= & \sum_{\substack{\left.\mathbf{x}_{\mathbf{j}}=\mathbf{x} \\
i \in \mathcal{I}_{r}(k) \backslash j\right\} \\
\mathbf{x}_{i} \in \tilde{\mathbf{C}}_{i}}} \frac{1}{\pi N_{0}} e^{-\frac{\mid y_{k}-\sum_{\left.i \in \mathcal{I}_{r}(k) h_{k}^{i} x_{k i}\right|^{2}}}{N_{0}}} \\
& \cdot \prod_{i \in \mathcal{I}_{r}(k) \backslash\{j\}} m_{u_{i} \rightarrow r_{k}}^{(\tau-1)}\left(\mathbf{x}_{i}\right),
\end{aligned}
$$

and

$$
m_{u_{j} \rightarrow r_{k}}^{(0)}(\mathbf{x})=1 / \tilde{M}_{j}, \quad \forall j \in \mathcal{J}, k \in \mathcal{I}_{u}(j), \mathbf{x} \in \tilde{\mathbf{C}}_{j} .
$$

Fig. 2 shows the decoding flow at the proposed pre-decoding assisted receiver. It is seen that there are two decision making modules. For the upper decision making module (marked in yellow), "Correct decoding" is decided based on the decoding results from all the $J$ users: "yes" implies that at least one user has decoded successfully, whereas "no" means no user has passed the CRC check.

Due to the iterative nature of the MPA, the reduced effective codebook size for one user may be interpreted as a reduction of the interference to other users. In this way, users can help each other to improve their error rate performance. As such, it is expected that DBIC-SCMA is capable of offering substantial gain over BIC-SCMA. To further improve the performance of DBIC-SCMA, a turbo-like combination of iterative detection and iterative decoding can be implemented similarly to the turbo-like receiver of the BIC-SCMA scheme [12].

2) Classical DBIC-SCMA Receiver Without Pre-decoding: A second class of DBIC-SCMA receiver, called "classical receiver", does not rely on pre-decoding and hence does not require the channel code to be systematic and rate-adaptive. The main difference with the proposed pre-decoding assisted

\footnotetext{
${ }^{5}$ This can be done by the introduction of CRC or by using channel codes with high error detection probability.
} 


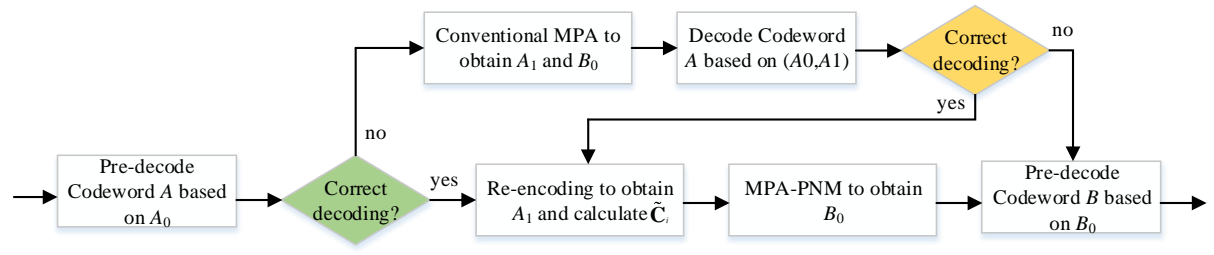

Figure 2. Decoding flow of the proposed pre-decoding assisted receiver.

receiver is that the classical receiver needs to receive all the sub-block groups related to a complete channel codeword before carrying out the decoding.

\section{Simulation Results}

We consider an SCMA system of 6 users sharing 4 resources. We use the SCMA codebooks of size $M=4[14]^{6}$ and the LDPC codes proposed for 5G-NR in 3GPP standard ${ }^{7}$. For the DBIC-SCMA system, we set $T_{0}=0$ and $T_{1}=1$. The parameters of the LDPC codes used in our simulations are the following: 1 ) $R=1 / 5$, frame length $=100$ bits; 2 ) $R=1 / 3$, frame length $=132$ bits; 3 ) $R=2 / 5$, frame length $=50$ bits. Since these 5G-NR LDPC codes are irregular, the natural block interleaving of DBIC-SCMA system allows us to take advantage of the bit-reliability (BR) mapping [15], [16] to facilitate the decoding.

Fig. 3 shows the average bit error rate (BER) and frame error rate (FER) performances in uplink Rayleigh fading channels for both BIC-SCMA and DBIC-SCMA. It can be observed that DBIC-SCMA achieves significant gains (e.g., at $R=2 / 5$, about $2.5 \mathrm{~dB}$ gain in terms of BER and FER) comparing to BIC-SCMA. Moreover, the curves show that the proposed pre-decoding assisted DBIC-SCMA receiver outperforms the classical DBIC-SCMA receiver. Fig. 4 shows the effect of Turbo iterations between the SCMA detector and the LDPC decoder on the error rate performance. The performances of both BIC-SCMA and DBIC-SCMA schemes are enhanced by employing a turbo-like receiver.

Fig. 5 shows the BER performance of all users in uplink Rayleigh fading channels, in which BIC-SCMA and DBICSCMA (with the proposed pre-decoding assisted receiver) are adopted by $N$ users and the remaining $6-N$ users, respectively, where $N \in\{0,3,6\}$. Note that $N=0$ corresponds to the case when all users employ DBIC-SCMA and $N=6$ corresponds to the case when all users employ BIC-SCMA. It can be observed that even when DBIC-SCMA is applied to a part of users, the BER performances of all users get improved. This is because the enhanced LLRs related to the coded bits of a certain user also help the remaining users sharing the same resources in the MPA decoding.

\section{CONCLUSIONS}

In this paper, we have proposed a novel DBIC-SCMA scheme with the principle of its transmitter and receiver, where

\footnotetext{
${ }^{6}$ Other codebooks could be used as well, e.g. [7].

${ }^{7}$ http://www.3gpp.org/ftp//Specs/archive/38_series/38.212/
}

the latter is based on pre-decoding of partially received signals by exploring certain LDPC codes with systematic and rateadaptive properties. We have shown that DBIC-SCMA offers substantial gains over the conventional BIC-SCMA scheme using short length LDPC codes for 5G-NR. The noticeable gains come from the fact that an improvement in the error rate performance of one DBIC-SCMA user helps boost the performance of the remaining ones.

It is noted that we have used MPA for SCMA detection. The complexity of the MPA detector grows exponentially with the maximum number of users sharing the same resource. As a future work, one may investigate the performance of DBICSCMA scheme using a low-complexity SCMA detector based on, for example, expectation propagation algorithm [17].

\section{REFERENCES}

[1] H. Ma, W. K. Leung, X. Yan, K. Law, and M. Fossorier, "Delayed bit interleaved coded modulation," in 2016 9th International Symposium on Turbo Codes and Iterative Information Processing (ISTC), Brest, France, Sep. 2016, pp. 86-90.

[2] D. Zhou, K. Niu, and C. Dong, "Delayed bit interleaved polar coded modulation," in 2018 IEEE 29th Annual International Symposium on Personal, Indoor and Mobile Radio Communications (PIMRC), Bologna, Italy, Sep. 2018, pp. 1-5.

[3] J. Du, Z. Wang, L. Xiao, L. Wang, and W. Qiao, "LDPC coded DBICM scheme for two way relay channel with a pipeline decoding structure," in 2018 IEEE International Conference on Communication Systems (ICCS), Chengdu, China, Dec. 2018, pp. 226-230.

[4] M. C. Lin and S. C. Ma, "A coded modulation scheme with interblock memory," IEEE Trans. Commun., vol. 42, no. 234, pp. 911-916, Feb. 1994.

[5] X. Yan, R. G. Machado, K. Huang, F. Gabry, M. Fossorier, H. Hafermann, H. Zhang, I. Land, and W. K. Leung, "Capacity analysis of delayed bit interleaved coded modulation," in 2018 IEEE 10th International Symposium on Turbo Codes Iterative Information Processing (ISTC), Hong Kong, Dec 2018, pp. 1-5.

[6] M. Vameghestahbanati, I. D. Marsland, R. H. Gohary, and H. Yanikomeroglu, "Multidimensinoal constellations for uplink SCMA systems - A comparative study," IEEE Commun. Surveys \& Tuts., vol. 21, no. 3, pp. 2169-2194, Third Quart. 2019.

[7] Z. Mheich, L. Wen, P. Xiao, and A. Maaref, "Design of SCMA Codebooks Based on Golden Angle Modulation," IEEE Transactions on Vehicular Technology, vol. 68, no. 2, pp. 1501-1509, Feb 2019.

[8] H. Nikopour and H. Baligh, "Sparse code multiple access," in IEEE 24th International Symposium on Personal Indoor and Mobile Radio Communications (PIMRC), London, UK, Sept. 2013, pp. 332-336.

[9] K. Yang, N. Yang, N. Ye, M. Jia, Z. Gao, and R. Fan, "Non-orthogonal multiple access: achieving sustainable future radio access," IEEE Commun. Magazine, vol. 57, no. 2, pp. 116-121, Feb. 2019.

[10] J. Bao, Z. Ma, M. Xiao, T. A. Tsiftsis, and Z. Zhu, "Bit-interleaved coded SCMA with iterative multiuser detection: multidimensional constellations design," IEEE Trans. Commun., vol. 66, no. 11, pp. 52925304, Nov. 2018. 


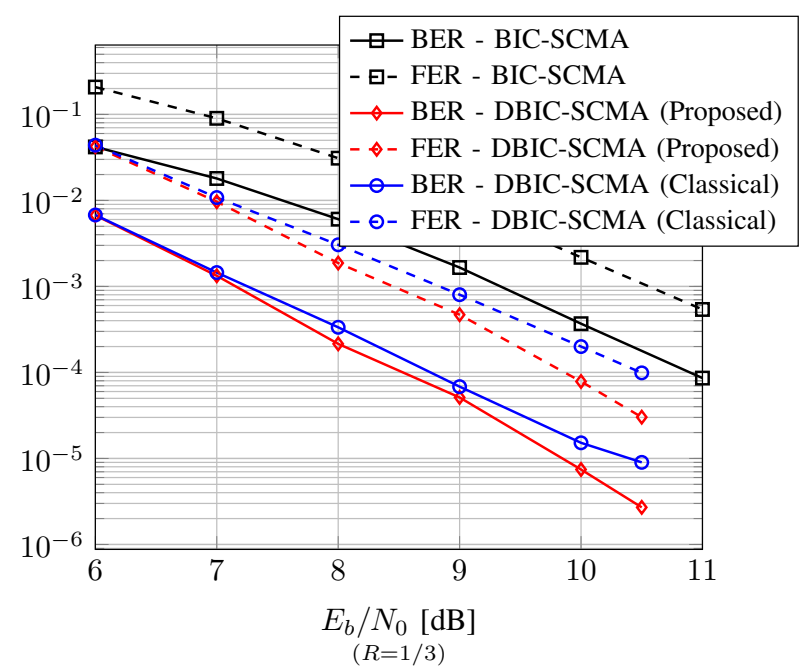

Figure 3. Average error rate performance in uplink.

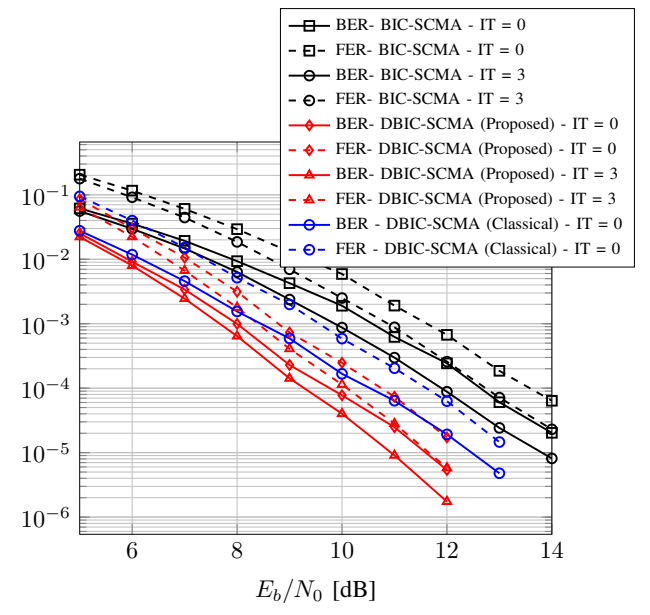

Figure 4. Uplink average error rate performance. $R=1 / 5$. IT denotes the number of turbo-iterations.

[11] B. Di, L. Song, Y. Li, and G. Y. Li, "TCM-NOMA: joint multiuser codeword design and detection in trellis-coded modulation-based NOMA for beyond 5G," IEEE J. Sel. Areas Commun., vol. 13, no. 3, pp. 766-780, Jun. 2019.

[12] B. Xiao, K. Xiao, S. Zhang, Z. Chen, B. Xia, and H. Liu, "Iterative detection and decoding for SCMA systems with LDPC codes," in 2015 International Conference on Wireless Communications Signal Processing (WCSP), Nanjing, China, Oct. 2015, pp. 1-5.

[13] R. Hoshyar, F. P. Wathan, and R. Tafazolli, "Novel low-density signature for synchronous CDMA systems over AWGN channel," IEEE Trans. Signal Process., vol. 56, no. 4, pp. 1616-1626, 2008.

[14] M. Taherzadeh, H. Nikopour, A. Bayesteh, and H. Baligh, "SCMA codebook design," in IEEE 80th Vehicular Technology Conference (VTC Fall), Vancouver, Canada, 2014, pp. 1-5.

[15] Z. Mheich, L. Wen, P. Xiao, and A. Maaref, "Unequal error protection SCMA codebooks," IEEE Trans. Veh. Technol., vol. 68, no. 4, pp. 40554058, Apr. 2019.

[16] Y. Li and W. E. Ryan, "Bit-reliability mapping in LDPC-coded modulation systems," IEEE Commun. Lett., vol. 9, no. 1, pp. 1-3, Jan. 2005.

[17] X. Meng, Y. Wu, Y. Chen, and M. Cheng, "Low complexity receiver for uplink SCMA system via expectation propagation," in 2017 IEEE Wireless Communications and Networking Conference (WCNC), Mar. 2017, pp. 1-5.
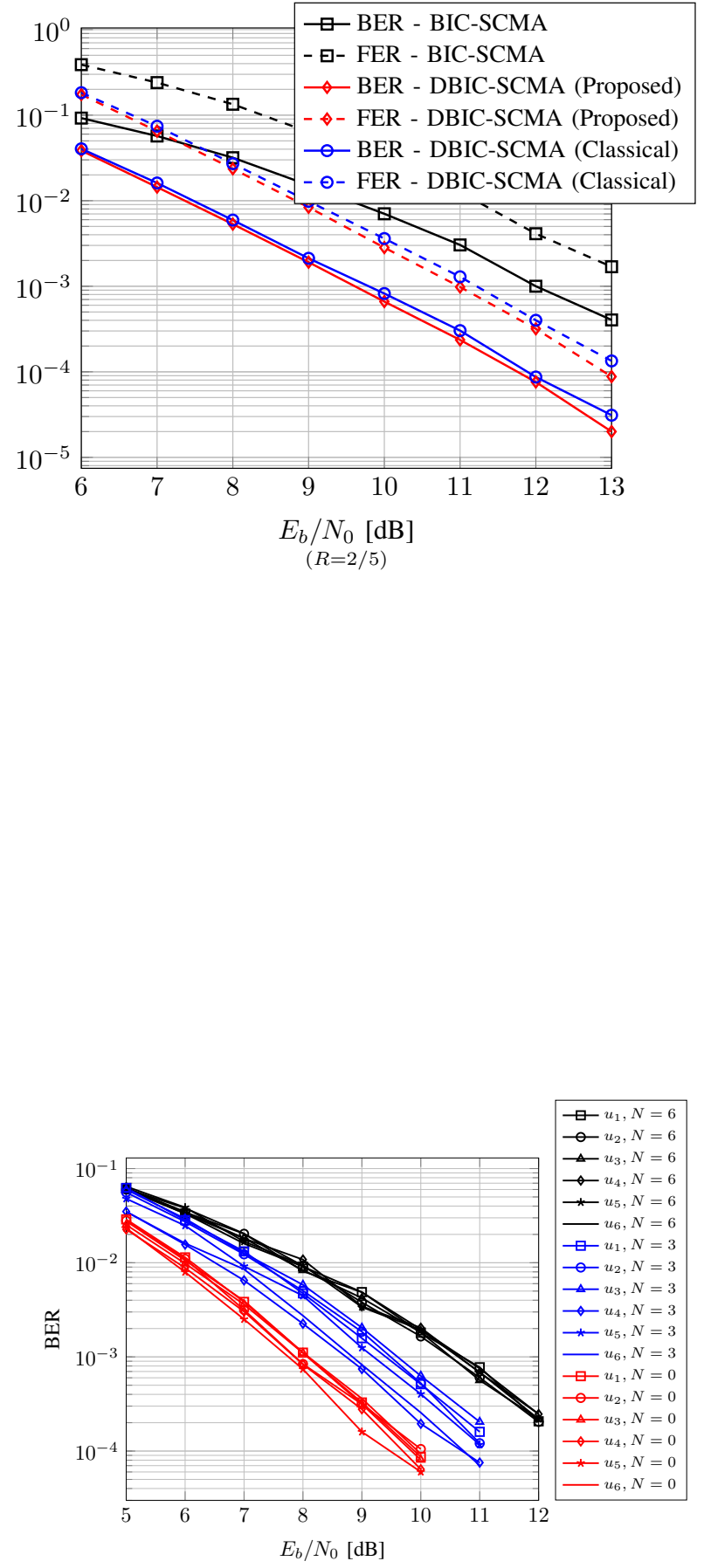

Figure 5. Uplink BER performance. $R=1 / 5 . N$ refers to the number of users using BIC-SCMA while the remaining users are using DBIC-SCMA (Proposed) 